\title{
PROPIEDAD MONÂSTICA Y SUSTENTO ALIMENTARIO: EL PATRIMONIO PRODUCTIVO DEL MONASTERIO DE SANT CUGAT DEL VALLÈS (SIGLOS X Y XI)
}

\author{
MARIA SOLER SALA
}

\author{
UDC: $27-788: 332(460.235) " 09 / 10 "$ \\ $726.71(460.235) " 09 / 10 "$ \\ Original scientific paper \\ Manuscript received: 01. 11. 2016. \\ Revised manuscript accepted: 27. 01. 2017. \\ DOI: 10.1484/J.HAM.5.113739
}

\author{
M. Soler Sala \\ Institute for Research on Medieval Cultures \\ University of Barcelona \\ c. Montalegre, 6 - o80o1 Barcelona, Spain \\ mariasoler@ub.edu
}

This paper 1 aims to study the productive assets of the monastery of Sant Cugat del Valles between the 10th and 11th centuries from a territorial perspective. Georeferencing the information in its rich cartulary and through the use of Geographic Information Systems (GIS), we intend to approach to the progressive increase of its possessions in the county of Barcelona and to the diversity of productive spaces (farmland, livestock, fisheries, food processing areas: mills and bread furnaces) that constituted their heritage. The maps resulting from this process will allow us to know the territorial evolution of the monastic property and their asset management strategies, designed to ensure both food sustenance of the monks and economic development of the community.

Keywords: Monastic landscape, productive assets, Benedictine economy, monastic diet, GIS.

\section{INTRODUCCIÓN}

Desde hace ya algunos años venimos interesándonos por el estudio territorial del fenómeno monástico medieval. Nuestra participación en la elaboración del Atlas CLAUS$T R A^{2}$ nos permitió comprender el interés de la georreferenciación en el análisis de las comunidades monásticas y su versatilidad para trabajar desde aproximaciones y perspectivas diversas ${ }^{3}$. Tales precedentes nos permitieron definir el concepto de paisaje monástico: una percepción sistémica del espacio monacal que se interesa no sólo por la trayectoria particular de una determinada comunidad, sino también por las relaciones que establece con su entorno inmediato, cercano o más lejano ${ }^{4}$. Todo ello, mediante el uso de sistemas de georeferenciación, conocidos como Geographical Information Systems (GIS), capaces de representar sobre cartografías históricas la diversidad de información que aparece en las fuentes.

Es a partir de esta perspectiva que pretendemos abordar el estudio sobre el impacto territorial de las fundaciones monásticas medievales, tanto en el espacio rural como en el entorno urbano. Así lo hemos hecho en un reciente análisis sobre el emplazamiento de las comunidades clarisas catalanas ${ }^{5}$, y así lo pretendemos hacer también en el estudio sobre la gestión patrimonial de algunos monasterios medievales.
Tal es el caso del cenobio de Sant Cugat del Vallès, que a través de un análisis minucioso de la documentación de los siglos X y XI nos permite conocer la distribución territorial de sus bienes productivos, así como las estrategias de gestión destinadas a garantizar el sustento alimentario de los monjes y el desarrollo económico de la comunidad.

\section{OBJETIVOS Y METODOLOGÍA}

Este estudio pretende analizar desde un punto de vista territorial la ubicación, diversidad y progresiva densificación del patrimonio productivo del monasterio de Sant Cugat del Vallès entre los siglos Xy XI. Con ello, conoceremos el impacto ejercido por el monasterio sobre los múltiples territorios situados bajo su jurisdicción. Nuestro objetivo consiste no solo en analizar los espacios destinados a la producción de alimentos (sobretodo, tierras de cultivo) sino también en conocer las relaciones que se establecen entre éstos y las necesidades alimentarias de la comunidad.

Desde el punto de vista espacial, el trabajo se enmarca en el ámbito del condado de Barcelona, donde el cenobio concentra la mayor parte de sus posesiones. Se trata de un territorio amplio, de casi $4.000 \mathrm{Km}^{2}$, con un de límite occidental variable en función del avance de la conquista condal. En su formulación definitiva se extiende desde las

\footnotetext{
${ }^{1}$ Este estudio forma parte de proyecto de investigación "Paisajes espirituales. Una aproximación espacial a las transformaciones de la religiosidad femenina medieval en los Reinos Peninsulares en la Edad Media (siglos XII-XVI)” (HAR2014-52198-P) dirigido por Blanca Garí y Núria Jornet (Universidad de Barcelona). ${ }^{2}$ El Atlas CLAUSTRA es una plataforma digital accesible a través de internet que cataloga y cartografía espacios de espiritualidad femenina medievales. Su desarrollo fue posible gracias al proyecto de investigación "CLAUSTRA. Atlas de espiritualidad femenina en los reinos peninsulares" (HAR2011-25127), dirigido por Blanca Garí (Universidad de Barcelona). Sus resultados pueden verse en URL: http://www.ub.edu/claustra.

3 Para un planteamiento teórico sobre la cuestión, véase B. GARÍ, M. SOLER, M. SANCHO et alii, CLAUSTRA. Propuesta metodológica para el estudio territorial del monacato femenino, en Anuario de Estudios Medievales 44, Barcelona, 2014, p. 21-50.

${ }^{4}$ Este es el objetivo del proyecto de investigación "Paisajes espirituales. Una aproximación espacial a las transformaciones de la religiosidad femenina medieval en los Reinos Peninsulares en la Edad Media (siglos XII-XVI)” (HAR2014-52198-P), dirigido por Blanca Garí y Núria Jornet (Universidad de Barcelona), cuyos primeros resultados pueden apreciarse en URL http://www.ub.edu/proyectopaisajes.

${ }_{5}$ X. COSTA, M. SANCHO, M. SOLER, Monacato femenino y paisaje. Los monasterios de clarisas dentro del espacio urbano en la Catalunya medieval, en Clarisas y Dominicas. Modelos de implantación, filiación, promoción y devoción en la Península Ibérica, Cerdeña, Nápoles y Sicilia. Firenze, Reti Medievali (en prensa).
} 
costas del Maresme hasta la marca del Penedès, pasando por las tierras del Vallès, la cuenca del Llobregat, la llanura barcelonesa y el territorio del Anoia. Dispone de una franja costera estrecha, prácticamente inexistente a la altura del Garraf pero amplia y con abundantes recursos en los deltas de los ríos Llobregat y Besòs. El condado de Barcelona queda limitado al norte por la línea que dibuja la sierra prelitoral, a través del macizo del Montseny, Sant Llorenç del Munt, Montserrat y la Sierra de Ancosa ${ }^{6}$.

Para un análisis como el que nos proponemos es necesario tener en cuenta todo tipo de fuentes a nuestro alcance: escritas, arqueológicas y territoriales. El estudio documental se ha realizado en base a la información contenida en el cartulario del monasterio de Sant Cugat del Vallès, publicado por José Rius a partir de una copia manuscrita del siglo XIII que se conserva en el Archivo de la Corona de Aragón 7 . Se trata de una colección de 1391 documentos que abrazan el período cronológico comprendido entre los años 904 y $1249^{8}$. Del conjunto de 778 documentos de los siglos Xy XI, hemos analizado con detalle aquellos que se corresponden con escrituras de adquisición de bienes (donaciones, legados o ejecuciones testamentarias, permutas, compra-ventas) por parte del monasterio.

La búsqueda de fuentes arqueológicas se ha realizado a partir del análisis de la Carta arqueológica del Servicio de arqueología de la Generalitat de Cataluña, y del vaciado de las memorias de excavación inéditas o publicadas resultado de las intervenciones realizadas en el marco cronológico y territorial estudiado. Nos han interesado especialmente las desarrolladas en espacios productivos de propiedad del monasterio 9 . Finalmente, el estudio territorial ha tenido en cuenta las características orográficas, edáficas e hidrográficas de los suelos del condado de Barcelona, así como su toponimia y microtoponimia. Esta última ha sido de gran utilidad en la difícil tarea de geoposicionar todas y cada una de las informaciones sobre la actividad productiva aparecidas en el Cartulario ${ }^{10}$.

El estudio de la información obtenida a través de las fuentes se ha realizado a través de un sistema integrado de bases de datos capaz de gestionar de manera uniforme informaciones de origen y procedencia distinta" ${ }^{11}$. La consideración de cada uno de los datos registrados como unidad informativa geográficamente coordenada nos ha permitido almacenar toda la información en una única base de datos y representarla sobre una misma cartografía digital. Para ello, hemos utilizado los Sistemas de Información Geográfica (GIS), que nos han permitido obtener una serie de mapas históricos sobre los bienes productivos (agrícolas, ganaderos y de transformación alimentaria) del monasterio. Tales cartografías, al igual que las obtenidas a partir de la interpolación cronológica o cuantitativa de los datos ${ }^{12}$ constituyen fuentes elaboradas a partir de las cuales articularemos nuestra interpretación (figs. 1 a 4).

\section{EL MONASTERIO DE SANT CUGAT DEL VALLÈS HASTA EL SIGLO XI}

El monasterio de Sant Cugat del Vallès fue, sin duda, el más importante del condado de Barcelona y uno de los más relevantes del territorio catalán medieval. Para cronologías de entre los siglos X y XI su interés no es solo económico, sino también político, por su papel en el proceso de consolidación de la conquista condal. A pesar de ello, y de poseer un extraordinario volumen de documentación escrita (en parte publicada) y numerosas campañas de excavación arqueológica a sus espaldas, todavía no dispone de una monografía completa sobre su evolución histórica ${ }^{13}$.

El monasterio se sitúa al pie de la sierra de Collserola, a unos quince quilómetros de la ciudad de Barcelona, en la

\footnotetext{
${ }^{6}$ La concreción minuciosa de los límites del condado ha sido definida por A. MAURI, La configuració del paisatge medieval: el comtat de Barcelona fins el segle XI, Tesis doctoral, Universidad de Barcelona, 2006, p. 6o-9o.

${ }^{7}$ J. RIUS, Cartulario de "Sant Cugat" del Vallés, 3 vols., Barcelona, 1945-1947. De aquí en adelante, las referencias a este repertorio documental se realizarán a través de la palabra Cartulario. A su vez, para la mención concreta de alguno de sus documentos se utilizará el acrónimo CSC, seguido del número de orden dentro del Cartulario y la fecha (año) de su redacción.

${ }^{8}$ En la publicación del Cartulario, J. Rius incluye como primer documento el conjunto de referencias que avalan la existencia de un precepto de confirmación de propiedades otorgado por Carlos el Calvo al monasterio en el año 875-877, el cual no se ha conservado.

${ }^{9}$ Tal es el caso, por ejemplo, de M. SOLER, S. REDONDO, A. MAURI, Memòria dels treballs d'investigació arqueològica al molí dels Monjos, Santa Margarida i els Monjos, Alt Penedès, Memoria de excavación inédita, Gelida, 2000.

${ }^{10} \mathrm{El} \mathrm{registro} \mathrm{y} \mathrm{geoposicionamiento} \mathrm{sistemático} \mathrm{de} \mathrm{los} \mathrm{datos} \mathrm{contenidos} \mathrm{en} \mathrm{la} \mathrm{documentación} \mathrm{escrita} \mathrm{fue} \mathrm{llevado} \mathrm{a} \mathrm{cabo} \mathrm{conjuntamente} \mathrm{por} \mathrm{A.} \mathrm{Mauri} \mathrm{y} \mathrm{M.}$ Soler durante la elaboración de sus respectivas tesis doctorales: A. MAURI, op. cit. (n. 6); M. SOLER, Els espais d'intercanvi. El mercat en el procés de gènesi i consolidació del feudalisme al comtat de Barcelona (segles IX a XIII), Tesis doctoral, Universidad de Barcelona, 2006.

"Ponemos aquí en práctica, aunque de manera adaptada, el sistema de trabajo diseñado por Alfred Mauri en su memoria de licenciatura y posteriormente enriquecido metodológica y conceptualmente en su tesis doctoral: A. MAURI, op. cit. (n. 6). Esta metodología ha sido utilizada en la realización de varios trabajos conjuntos (A. MAURI, M. SOLER, Les conques baixes del Llobregat i l'Anoia a la fi del primer mil.leni, en Catalunya i Europa a la fi del Primer mil.leni, Congrés Internacional Gerbert d'Orlhac i el seu temps (Vic-Ripoll, 10-13 de novembre de 1999), Vic, 1999, p. 209-225; A. MAURI, M. SOLER, Els castells i l'organització i explotació del territori a la línia del Llobregat i el Penedès (segles IX-XI), en Els castells a la Mediterrània nord occidental, Actes del congrés (Arbúcies, 5-7 de marc de 2003), Arbúcies, 2004, p. 131-140 así como en la elaboración de la tesis doctoral de la autora del presente estudio: M. SOLER, op. cit. (n. 10).

${ }^{12}$ La interpolación de datos constituye una útil herramienta de representación cartográfica que permite crear modelos evolutivos a partir de los datos almacenados en nuestra base de datos. El mapa 2 de la fig. 1, lo hace a partir de la fecha de cada unidad de información registrada, otorgando una coloración fría (azul) a las cronologías más antiguas, y colores cálidos (rojo) a las más modernas. El mapa 3 de la fig. 1 y los mapas 2 y 4 de las figs. 2 y 3 constituyen, en cambio, representaciones sobre la densidad de información, siendo las coloraciones rojas las que concentran un mayor número de menciones documentales. ${ }^{13}$ El interés por el monasterio de Sant Cugat del Vallès se ha centrado más en el estudio del conjunto arquitectónico que en la trayectoria histórica de la comunidad. Destacan, en este sentido: J. AMBRÓS, El monestir de Sant Cugat del Vallès, Barcelona, 1985; M. BOFILL, El monasterio de Sant Cugat del Vallès. Guía histórica y arquitectónica, Barcelona, 1994; o también P.Ll. ARTIGUES, El monestir de Sant Cugat del Vallès. L'evolució arquitectònica a partir de l'arqueologia, en II Congrés d'Arqueologia Medieval i Moderna a Catalunya, Sant Cugat del Vallès, 18-21 d'abril de 2002, vol. I, Sant Cugat del Vallès, 2003, p. 18-28. Desde un punto de vista histórico, son interesantes dos estudios relativos al origen y formación de su dominio patrimonial. El primero, de manera extensa: J.M. SALRACH, Formació, organització i defensa del domini de Sant Cugat en els segles X-XII, en Acta historica et archaeologica mediaevalia 10, Barcelona, 1992, p. 127-173; y el segundo, acotando el estudio al territorio del Vallès durante la décima centúria: X. BOU, El domini del Monestir de Sant Cugat durant el segle X al Vallès, Tesis de licenciatura, Universidad de Barcelona, 1985.
} 
actual comarca del Vallès Occidental. De acuerdo con los documentos de su Cartulario, se emplazaba "in comitato Barchinona, in locum vocitatur Octaviano ${ }^{14}$, un topónimo de origen imperial atribuido a la existencia de un castrum de época romana ${ }^{15}$. Ya en el IV, se conoce la construcción de un martyrium funerario, donde debieron depositarse los restos de San Cucufate (Sant Cugat, en catalán), de quien la tradición afirma que sufrió martirio en este lugar ${ }^{16}$. Entre el final de la quinta centuria y el inicio de la sexta, dicha estructura y el aula rectangular adosada a ella fueron destruidas por un incendio. A pesar de ello, el lugar no se abandonó y a la estructura reconstruida se le añadió un ábside, convirtiéndose así en basílica visigoda ${ }^{17}$. La localización de numerosos silos en las inmediaciones del templo ha permitido interpretar no sin discusión - la existencia de una primera comunidad religiosa entre finales del siglo VI y principios del siglo $\mathrm{VII}^{18}$.

Poco sabemos sobre las vicisitudes ocurridas en este espacio en época musulmana, más allá de su destrucción en el año $780^{19}$. Es posible que la vida espiritual del lugar no se reanudara hasta después del año 801, con la conquista franca de Barcelona. Sin embargo, el primer testigo documental sobre su existencia no llegará hasta el año 878 , a través de un precepto real de confirmación de bienes a la catedral de Barcelona ${ }^{20}$. La tranquilidad duraría bien poco: el embate de Almanzor sobre tierras catalanas en el 985 supondría la destrucción de la abadía, la desaparición del archivo y la muerte de los monjes ${ }^{21}$. Tales circunstancias sumieron al monasterio en una ardua tarea de reconstrucción. La venta de varias posesiones por parte del abad en el año 1013 tenía como objetivo la restitución del claustro, del cual quedan algunos vestigios en el subsuelo ${ }^{22}$. Sin embargo, de la iglesia que se estaba construyendo a mitad de la onceava centuria no nos queda más testigo que su campanario ${ }^{23}$. Tales estructuras serían substituidas por la iglesia y los claustros que empezaron a construirse a mediados del siglo XII y que han llegado hasta nosotros ${ }^{24}$.

Mientras todo ello acontecía, el monasterio supo articular un rico y amplio patrimonio sobre el que ejerció su jurisdicción. Tal acumulación de propiedades fue posible gracias a la recepción de múltiples donaciones (piadosas o testamentarias) y mediante algunas (menos) permutas y compra-ventas directas de bienes ${ }^{25}$. La georeferenciación de las propiedades documentadas en el Cartulario sobre el mapa (fig. 1, mapa 1) demuestra que a finales del siglo XI el monasterio disponía de un sólido patrimonio distribuido a lo largoy ancho del condado de Barcelona. Se articulaba en base a tres grandes espacios territoriales: el Vallès, la cuenca del Llobregat y el llano de Barcelona, y las recién incorporadas tierras del Anoia y del Penedès.

La interpolación cronológica de los datos ${ }^{26}$ (fig. 1, mapa 2) nos permite comprobar que el núcleo patrimonial original (coloraciones azuladas) se encontraba en el territorio del Vallès, donde se ubican más de la mitad $(51,24 \%)$ de las referencias registradas. Este territorio de suelos fértiles y rica hidrografía favoreció el desarrollo de una próspera agricultura. Tal concentración de bienes (fig. 1, mapa 3) no se produjo solo en el espacio más inmediato al monasterio (1040 menciones), sino también, y sobretodo, en las tierras adyacentes a las cuencas de los ríos Ripoll, Congost y Mogent (1295), así como en el territorio de Cardedeu (169) y Palautordera (89). Más al norte, el monasterio acumulaba propiedades en las inmediaciones de Caldes de Montbui (150), Terrassa (47) y Castellar del Vallès (43).

La fértil llanura barcelonesa y la cuenca del río Llobregat disfrutaron también del interés del monasterio. En ellas se concentra el 25,36\% de las menciones registradas durante los siglos Xy XI. En este espacio destacan los predios adquiridos en las inmediaciones de la ciudad de Barcelona (355), así como los bienes ubicados en el delta del río Llobregat (295). En la margen derecha de dicho curso hidráulico, el monasterio concentró numerosas propiedades en el término del castillo de Cervelló (255), sobre el que ejerció su jurisdicción ${ }^{27}$, y en el territorio de Eramprunyà (248). Cerca de este último se fundó el monasterio de Santa María de Castelldefels, que entre los años 967 y 977 dependió del monasterio de Sant Cugat ${ }^{28}$.

La progresión del dominio monástico sobre las tierras del Anoia y el Penedès estuvo íntimamente relacionada

\footnotetext{
${ }_{14}$ Así lo citan, por ejemplo, los documentos CSC, 2, año 904; CSC, 15, año 938; CSC, 34, año 953; CSC, 37, año 955; CSC, 43, año 956; CSC, 50, año 957 y CSC, 57, año 959, entre muchos otros.

${ }^{15}$ J. AMBRÓS, op. cit. (n. 13), p. 10-14.

${ }^{16}$ Tanto el poeta hispanorromano Prudencio como los antiguos libros de liturgia hacen referencia al mártir Cucufate, víctima de las persecuciones contra el cristianismo acontecidas en el territorio del imperio a principios del siglo IV. M. BOFILL, op. cit. (n. 13), 13-14.

${ }^{17}$ X. BARRAL, La basilique paléochrétienne et visigotique de Sant Cugat del Vallès (Barcelone). Dossier archéologique et essai d'interprétation, en Mélanges de l'Ėcole française de Rome. Antiquité 86, Rome, 1974, p. 891-928.

${ }^{18}$ Así lo hipotetizaron, en los años sesenta, P. Bosch i Gimpera y J. de C. Serra Ràfols. Sin embargo, autores más recientes como J.A. Adell, E. Riu-Barrera y M. Blasco consideran que no existen suficientes indicios para poder considerar la existencia de esta primera comunidad. P.Ll. ARTIGUES, op. cit. (n. 13), p. 20. ${ }^{19}$ En opinión de J.M. Salrach, el infructuoso intento del abad de Saint-Denis de trasladar las reliquias de San Cucufate en el año 778 constituye un buen indicio sobre la continuidad del culto en época musulmana. J.M. SALRACH, op. cit. (n. 13), p. 128.

${ }^{20}$ Poco sabemos sobre el monasterio del siglo IX. A nivel arquitectónico, es posible que fuera el resultado de un proceso de reaprovechamiento de materiales y estructuras de época anterior. De él ha pervivido solamente un muro en el claustro actual, con ocho ventanas y una puerta. J. AMBRÓS, op. cit. (n. 13), p. 23.

${ }^{21}$ A. PLADEVALL, F. CATALÀ-ROCA, Els monestirs catalans, Barcelona, 1970, p. 220.

${ }^{22}$ CSC, 456, año 1013 .

${ }^{23}$ CSC, 629, año 1063; CSC, 632, año 1063. M. BOFILL, op. cit. (n. 13), p. 15.

${ }^{24}$ A. PLADEVALL, op. cit. (n. 21), p. 220.

${ }^{25}$ El minucioso estudio elaborado por J.M. Salrach en relación al proceso de adquisición de propiedades por parte del monasterio nos permite conocer la proporción de donaciones $(82,33 \%)$ y compras $(17,67 \%)$ durante los siglos X y XI. J.M. SALRACH, op. cit. (n. 13), p. 137-152.

${ }^{26}$ Para más detalles sobre las herramientas de interpolación cartográfica, véase supra nota 12.

${ }^{27}$ J.M. SALRACH, op. cit. (n. 13), p. 150.

${ }^{28} \mathrm{X}$. BOU, op. cit. (n. 13), p. 20.
} 
con el proceso de conquista y consolidación de la denominada marca extrema, situada más allá de la frontera del río Llobregat. Entre ambos espacios se concentra el 23,40\% de las menciones (7,29\% en el Anoia y 16,11\% en el Penedès), algunas de ellas de cronología muy temprana (fig. 1, mapa 2). El dominio del monasterio sobre este espacio se articuló entorno a las principales fortificaciones construidas en el territorio, utilizadas como punto de lanza en el proceso de conquista condal: Olèrdola (412) y Subirats (79) en el Penedès, y Masquefa (309) en el Anoia, constituyen buenos ejemplos de ello. El monasterio poseía la jurisdicción sobre este último castillo y también sobre el de Clariana, además de la que ejercía sobre los castillos de Albiñana, Sant Vicenç, Calders y Santa Oliva, en tierras del Penedès ${ }^{29}$.

Así pues, a finales del siglo XI, el monasterio de San Cugat disponía de un amplio patrimonio. Ejercía su dominio sobre tierras y campesinos y poseía la jurisdicción sobre múltiples castillos, iglesias y monasterios. Se había convertido ya en un poderoso agente feudalizador del territorio.

\section{DIVERSIDAD DE ESPACIOS PRODUCTIVOS}

La mayor parte de las propiedades que constituían el patrimonio del monasterio estaban dedicadas a la producción de alimentos. Eran sobretodo tierras agrícolas, destinadas al cultivo de cereal y viña, así como a la plantación de verduras, legumbres, frutas y hortalizas.

Sin duda, de entre todas las especies cultivadas destaca el predominio del cereal. Las fuentes estudiadas documentan una numerosa cantidad de referencias a campo, champo o kampo, que junto a una parte importante de las terrae constituyen menciones indirectas al cultivo de grano ${ }^{30}$. Las concentraciones de tierras de cereal son abundantes en los tres ámbitos que configuran su dominio patrimonial (fig. 2, mapas 1 y 2). Se detecta mayor densificación en el espacio inmediato al monasterio (525) y en las tierras que flanquean la confluencia de los ríos Ripoll, Congost y Mogent (331). Son abundantes también en la cuenca baja del río Llobregat (187), así como en tierras de Cervelló (279) y Eramprunyà (140). En el espacio del Anoia, registramos concentración de campos de cereal en Masquefa (85), y en el Penedès, en los alrededores del castillo de Olèrdola (150) y Subirats (38). Si bien es cierto que el trigo ${ }^{31}$ era el cereal más apreciado, observamos una amplia difusión de la cebada ${ }^{32}$, que por ser menos exigente y más barata se convirtió en uno de los cultivos más abundantes.
La segunda especie en profusión de menciones es la vid, con un $27,80 \%$ del total de referencias agrícolas registradas. La distribución territorial de la viña (fig. 2, mapas 3 y 4) nos permite detectar la existencia de dos principales espacios de concentración: la confluencia de los ríos Ripoll, Congost y Mogent (321) y el llano de Barcelona (166). En el primer caso, observamos la creación de un espacio especializado en la producción vitícola que prefiere la calidad de las tierras ubicadas al este del monasterio que los predios dispuestos alrededor del mismo (123). Tal concentración se detecta no sólo a través de la densidad de menciones, sino también por el hecho que en la mayoría de casos las viñas sean colindantes unas con otras, conformando vinearios como los de Corró y Cerdanyola ${ }^{33}$. Por su lado, la concentración de espacios vitícolas detectada en el llano barcelonés y en la cuenca baja del Llobregat (con un total de 257 menciones) está relacionada con el denominado hort y vinyet de Barcelona, un ámbito de especialización agrícola destinado al abastecimiento de fruta, verdura, productos frescos y vino a la ciudad ${ }^{34}$. Más allá del curso del Llobregat, en tierras del Penedès, destaca Olèrdola (113), que documenta también un rico vineario propiedad del monasterio ${ }^{35}$. Importantes concentraciones de viñas se detectan también en Masquefa (50), Cervelló (49) y Eramprunyà (49), así como alrededor de algunas villas mercado vallesanas como Caldes de Montbui (44), Cardedeu (43) y Terrassa (16).

Los huertos constituyen un 5,6\% del total de referencias agrícolas registradas en el Cartulario (fig. 3, mapas 1 y 2). Eran abundantes en el entorno del monasterio y de los cursos fluviales del Vallès, alrededor del río Besòs y de su red hidráulica subsidiaria: el río Ripoll, la riera de Caldes y los ríos Tenes, Congost y Mogent (34). Las menciones de huertos son numerosas también en las llanuras barcelonesas y en el delta del río Besòs (36 menciones en total), espacios muy vinculados al mencionado hort i vinyet de la ciudad. Más allá de Barcelona y del Vallès, la huerta es importante también en el territorio de marca más inmediato al río Llobregat, con concentraciones significativas en los términos de los castillos de Cervelló (14) y Eramprunyà (13). En el Anoia y el Penedès destacan las concentraciones documentadas en Masquefa (33) y Olèrdola (13), respectivamente. Dada la necesidad de disponer de recursos hídricos suficientes, en todos estos espacios se detecta la presencia de una extensa red de canales de riego capaces de conducir el agua procedente de torrentes, ríos y rieras a los campos de cultivo ${ }^{36}$. Lamentablemente, la documentación estudiada es poco explícita en cuanto a la

\footnotetext{
${ }_{29}^{2}$ J.M. SALRACH, op. cit. (n. 13), p. 139-140, 149-150.

$3^{30}$ Tal interpretación la sostienen también autores como J.M. SALRACH, op. cit. (n. 13), p. 138. Si consideramos que la mayor parte de las "tierras" sin definición concreta de cultivo se dedicaban al cereal, éstas supondrían un 56,9\% del total de los bienes agrícolas documentados.

${ }^{31}$ CSC, 377, año 1002; CSC, 431, año 1010; CSC, 537, año 1032; CSC, 559, año 1042, entre muchas otras referencias.

${ }^{32}$ Las referencias al cultivo de cebada son muy numerosas. Así lo vemos, por ejemplo, en aquella "ciga I plena de ordeo" (CSC, 188, año 986), o en otros múltiples documentos: CSC, 56, año 958; CSC, 101, año 973; CSC, 171, año 985; CSC, 188, año 986.

33 CSC, 159, año 984; CSC, 617, año 1059.

${ }^{34}$ Tal es el caso de los "vinearios" documentados en CSC, 333, año 998 y CSC, 650, año 1066. Sobre la importancia económica de este espacio productivo, véase PH. BANKS, L'estructura urbana de Barcelona, 714-130o, en Història de Barcelona, vol. II, Barcelona, 1992, p. 25-71; y también J. SOBREQUÉS, S. RIERA, Els orígens medievals del territori de Barcelona, en L’Avenç 88, Barcelona, 1985, p. 11-13.

35 CSC, 537, año 1032

${ }^{36}$ Sobre la importancia y configuración de la infraestructura de irrigación del Vallès, véase J. VILAGINÉS, El paisatje, la societat i l'alimentació al Vallès Oriental (segles X-XII), Abadia de Montserrat, 2001, p. 98-99. Para un análisis territorial de los espacios irrigados cercanos al monasterio (Sant Cugat, Rubí, Cerdanyola i Ripollet), véase H. KIRCHNER, Espais agraris en el terme del monestir de Sant Cugat del Vallès (segles X-XIII), en Arqueologia medieval 2, 2006, p. 22-35.
} 
variedad de productos hortícolas cultivados y no nos permite conocer más especies que las habas ${ }^{37}$ y los guisantes ${ }^{38}$. Sin embargo, la diversidad de productos debió ser bastante mayor: legumbres como las lentejas y los garbanzos, así como verduras como la col, la cebolla, el ajo, el puerro, el nabo, la acelga y la calabaza debieron ser abundantes en los huertos propiedad del monasterio 39 .

Tanto en los huertos como en algunas viñas y en otras tierras de cultivo se detecta una práctica abundante de la arboricultura $^{40}$, que dispone de un $8,85 \%$ de las menciones documentadas (fig. 3, mapas 3 y 4). Su distribución geográfica está muy relacionada con la de los huertos, puesto que en la mayoría de casos el cultivo de frutales se realizaba en su interior. De acuerdo con ello, se detecta una especial concentración de los mismos tanto en las muy irrigadas tierras del Vallès (78), como en los dominios de Masquefa (57) y Olèrdola (57). De igual manera, no podemos dejar de mencionar la densidad de referencias detectadas en los llanos barceloneses y el delta del río Besòs (25), relacionadas también con el hort $i$ vinyet barcelonés. Al igual que en el resto de tipos agrícolas estudiados, la documentación es poco explícita en cuanto a las especies cultivadas. Distingue entre dos tipologías: los pomiferos (fruta dulce) y los impomiferos (fruta seca). Prácticamente la mitad de las menciones documentadas se refieren a árboles de fruto seco como los belloteros, castaños o nogales, cuyos frutos se utilizaban sobretodo para el alimento de los animales, aunque podían consumirse en tiempos de escasedad ${ }^{41}$. De la otra mitad solo se nos especifica la presencia de higueras ${ }^{42}$, manzanos $^{43}$, cerezos $^{44}$ y melocotoneros ${ }^{45}$.

Vinculados tanto a la extensión del cultivo de cereal como a la generalización del consumo de pan, documentamos la presencia de una gran cantidad de los molinos hidráulicos (104). Tal y como puede apreciarse en el mapa (fig. 4, mapa 4), eran abundantes en todo el dominio santcugatés, pero muy especialmente alrededor del espacio fluvial constituido por los ríos Besòs, Congost y Mogent. Destacan aquí por su cantidad los molinos adquiridos alrededor río Ripoll, donde probablemente el monasterio creó un espacio de especialización harinera ${ }^{46}$. Muy cerca de este ámbito, el monasterio disponía de molinos en la riera de Rubí, en la Tordera, en Riusec y en la riera de Caldes. También en los territorios de las villas de Sabadell y Terrassa. En el espacio fluvial del Llobregat se detectan molinos en Cervelló y Castelldefels, así como en el territorio de Barcelona. En el Penedès, documentamos molinos en Torrelavit, Subirats y Olèrdola, y ya en el Anoia, también en Masquefa. Entre todos ellos, hay uno que conocemos bien: el denominado Molí dels Monjos, en el término actual de Santa Margarida i els Monjos, localizado a partir de dos documentos del Cartulario y objeto de un interesante proceso de excavación arqueológica y musealización ${ }^{47}$.

Tal profusión de menciones de molinos contrasta con la práctica inexistencia de referencias a hornos de pan $^{48}$ tal vez porqué en este momento no suponían todavía un recurso económico significativo. En las descripciones de los alodios objeto de adquisición por parte de los monjes aparecen también numerosos bosques y yermos. Los primeros ofrecían una magnífica reserva de productos alimentarios tanto para los campesinos cultivadores de las tierras, como para el abastecimiento del monasterio. Los segundos, en cambio, junto con los garricis y los prata et pasqua aparecidos en los documentos constituían extensas áreas de pastoreo para el ganado (fig. 4, mapa 1). Prácticamente todas las unidades de explotación descritas por las fuentes disponían de pequeños establos o corrales destinados a la cría de un pequeño contingente deanimales. El Cartulario nos permite documentar una gran variedad de ellos: como cerdos (123), ovejas (308) y cabras (240), así como volatería diversa: gallinas (11), patos (8), gansos (37), capones (5) y palomares para la reproducción de palomas (11). Las referencias a bueyes (49) y vacas (39) son abundantes, así como a équidos utilizados para el transporte: como el caballo (10), la yegua (23), la mula (40), la somera (11) y el asno (21).

\section{RELACIÓN CON LAS VILLAS MERCADO}

El proceso de sucesiva ampliación del patrimonio del monasterio sugiere que durante los siglos X y XI disfrutó de una próspera economía. Por aquel entonces, la comunidad de monjes vivía de las rentas generadas por sus dominios, tanto a través del cobro de partes de cosecha (el diezmo y la tasca, sobretodo) como de censos en moneda. A tales beneficios se le sumaban diversas cargas eclesiásticas (diezmos, primicias y oblaciones), derechos jurisdiccionales (como el de impartir justicia) y los rendimientos obtenidos a través de la explotación de sus propias dominicaturas ${ }^{49}$.

La distribución de sus propiedades productivas sobre el territorio condal y su concentración en áreas coherente-

37 CSC, 443, año 1012.

${ }^{38}$ CSC, 467, año 1018.

39 A. RIERA-MELIS, Senyors, monjos i pagesos: alimentació i identitat social als segles XII i XIII, Barcelona, 1997, p. 26.

$4^{\circ}$ La documentación vincula la presencia de árboles frutales no sólo con los espacios de huerto, sino también con las viñas. Así lo vemos en las múltiples referencias a "vineas cum arboribus". CSC, 17, año 939; CSC, 243, año 990; CSC, 263, año 991; CSC, 283, año 992; X. BOU, op. cit. (n. 13), p. 52.

${ }^{41}$ J. VILAGINÉS, op. cit. (n. 36), p. 83.

${ }^{42}$ CSC, 130, año 978; CSC, 188, año 986; CSC, 212, año 987; CSC, 393, año 1004.

${ }^{43}$ CSC, 41, año 955; CSC, 43, año 956; CSC, 71, año 964, entre muchas otras.

${ }_{44}$ CSC, 427, año 1010.

45 CSC, 495, año 1024

${ }^{46}$ X. BOU, op. cit. (n. 13), p. 70; J.M. SALRACH, op. cit. (n. 13), p. 139; H. KIRCHNER, op. cit. (n. 36), p. 22-35.

${ }_{47}$ CSC, 130, año 978; CSC, 134, año 979. Sobre la extensa evolución histórica del molino, véase M. SOLER, S. REDONDO, A. MAURI, Memòria dels treballs d'investigació arqueològica al molí dels Monjos, Santa Margarida i els Monjos, Alt Penedès (Memoria de excavación inédita), Gelida, 20oo; A. MAURI, M. SOLER, El molí del Foix: un exemple d'intervenció integral en el patrimoni històric edificat, en I Trobada d'Estudiosos del Foix, Castellet i la Gornal, Santa Margarida i els Monjos, 4-6 de novembre de 2004, Barcelona, 2005, p. 205-210.

${ }^{48}$ Hemos localizado solo uno (CSC, 377, año 1002) utilizado como elemento de afrontación con un huerto cedido al monasterio por vía de testamento.

49 J.M. SALRACH, op. cit. (n. 13), p. 140, 148. 
mente organizadas sugiere que la adquisición de bienes no fue fruto de la casualidad (como podría considerarse por el carácter supuestamente aleatorio de las donaciones) $)^{50}$ sino de una estrategia económica bien definida, interesada en crear espacios de concentración agrícola en lugares de elevado rendimiento económico. No sólo desde el punto de vista de la fertilidad de los suelos, sino también por su proximidad con las plazas de mercado ${ }^{51}$.

Por desgracia, las fuentes escritas disponibles son poco explícitas en este sentido y no permiten analizar esta cuestión con el detalle al que ha podido llegar Paul Devroey en el caso de las las grandes abadías carolingias francesas, las cuales sabemos que mantenían una estrecha relación con el mercado. Para estos monasterios, los mercados constituían espacios de elevado interés económico: de ellos obtenían productos de los que no disponían (sal, vestido y alimentos en épocas de escasez) pero en él vendían también sus excedentes agrícolas con el objetivo de garantizar el sustento económico de la comunidad ${ }^{2}$.

Nuestra percepción sobre el interés de Sant Cugat del Vallès por el mercado es siempre indirecta y se basa en tres elementos fundamentales. Por un lado, las numerosas menciones en el Cartulario tanto de mercados semanales, como del sistema de pesos y medidas utilizado en cada uno ellos, convierte este lugar en un espacio conocido, nada extraño para los monjes. Gracias a tales referencias, siempre indirectas, conocemos la existencia de los mercados semanales más antiguos del condado de Barcelona: Martorell53, Caldes de Montbuii ${ }^{54}$, la Granada ${ }^{55}$, Sabadell ${ }^{56}$ y también Sant Cugat del Vallès ${ }^{57}$, que además de mercado dispondría (ya en el siglo XII) de una de las ferias más antiguas del territorio catalán ${ }^{5}$. El hecho de que tanto el mercado como la feria se celebraran cerca del edificio monástico deja constancia de la intensa relación del monasterio con este espacio.

En segundo lugar, por la especialización de cultivos de sus tierras de labranza. La obsesión por generalizar el conreo de cereal y viña (que en su conjunto significa un $85 \%$ del total de menciones documentadas) nos habla no solo de la importancia de dos productos (el pan y el vino) importantes para el consumo interno de la comunidad, sino también de dos mercancías poderosas con las que comerciar en el mercado $^{59}$. En un contexto de ampliación del espacio cultivado e incremento de la producción agrícola como el vivido en los siglos X y XI en el condado de Barcelona, el mercado se convierte en una válvula fundamental para la comercialización del excedente agrario ${ }^{60}$. Tal objetivo era necesario para todos. Lo era para los campesinos que trabajaban las tierras del monasterio, que utilizaban el mercado para intercambiar sus excedentes por moneda o por productos que no disponían. Pero también debió serlo para el monasterio, que con la comercialización del cereal y el vino procedente de sus rentas podría obtener nuevos recursos para el sustento de la comunidad.

En tercery último lugar, el interés de Sant Cugat del Vallès por el mercado lo detectamos también a partir del análisis territorial de las preferencias de los monjes en la adquisición de tierras de cultivo (sobretodo viñas y huertos) en entornos circundantes a las villas mercado. Como se ha podido observar, el monasterio disponía de abundantísimas propiedades agrícolas no sólo en el denominado hort i vinyet de Barcelona, sino también alrededor de las principales villas del condado barcelonés como Granollers (181), Caldes de Montbui (149), Cardedeu (100), Terrassa (65), Sant Celoni (56), Sabadell (28) o la misma Sant Cugat del Vallès, todas ellas con celebración de mercado semanal documentado a lo largo del siglo XI. Tal proximidad podría darnos a conocer no solo la participación del monasterio en las transacciones económicas que en ellos se desarrollaban, sino incluso también, como se ha demostrado en el caso de las grandes abadías francesas ${ }^{61}$, el importante papel jugado por la producción monástica en el aprovisionamiento de algunas de estas villas medievales.

\section{LA ALIMENTACIÓN DE LOS MONJES}

La documentación escrita del Cartulario nos ofrece muy poca información sobre la dieta de los monjes santcugatenses. Para los siglos Xy XI, a diferencia de lo que ocurrirá posteriormente $^{62}$, no disponemos de fuentes directas capaces

\footnotetext{
$\overline{5^{\circ}}$ El análisis porcentual de los datos obtenidos por J.M. Salrach en este sentido nos permite detectar un amplio predominio de las donaciones (82,33\%) por encima de las compras (17,67\%). Véase, supra nota 25.

${ }^{51}$ Sobre la progresiva articulación de la red de mercados rurales del condado de Barcelona, véase M. SOLER, op. cit. (n. 10); M. SOLER, From «forum Granate» to Vilafranca's fair. Origin, foundation and articulation of a market network in the Feudal Penedès (9th-12th c.), en SVMMA. Revista de Cultures Medievals 6, 2015, p. 171-19o; M. SOLER, Territorio e intercambios. Origen, transformación y consolidación de la red de mercados rurales del condado de Barcelona entre el siglo XI y la primera mitad del siglo XIV, en Actas del Congreso Internacional Industrias y mercados rurales en los reinos hispánicos (siglos XIII-XV), Zaragoza, 28-30 de junio de 2016, Zaragoza (en prensa).

${ }^{52}$ J. P. DEVROEY, Un monastère dans l'économie d'échanges: les services de transport à l'abbaye Saint-Germain-des-Prés au IXe siecle, en Annales. Économies, sociétés, civilisations 39, 1984, p. 570-589; J. P. DEVROEY, Production et circulation dans un domaine monastique à l'èpoque carolingienne: l'exemple de l'abbaye de Saint-Denis, en Revue belgue de philologie et d'histoire 75, 1997, p. 943-945.

53 CSC, 524, año 1032; CSC, 529, año 1033.

${ }_{54}$ CSC, 564 , año 1043.

55 CSC, 699, año 1080.

${ }^{56}$ CSC, 821, año 1113 .

57 CSC, 674, año 1072.

${ }^{58}$ C. BATLLE, Fires i mercats. Factors de dinamisme econòmic i centres de sociabilitat (segles XI a XV), Barcelona, 2004, p. 183.

${ }^{59}$ A. RIERA-MELIS, M. SOLER, La distribución y los mercados de alimentos en el Mediterráneo Occidental (siglos VIII-XII), en L'Alimentazione nell'alto medioevo: pratiche, simboli, ideologie, LXIII Settimane di studio della Fondazione Centro di Studi sull'Alto Medioevo (Spoleto, 9-14 aprile de 2015), Spoleto, 2016, p. 213-298.

${ }^{60}$ M. SOLER, op. cit. (n. 10), p. 419-421.

${ }^{61}$ J.P. DEVROEY, op. cit. (n. 52), 1984, p. 571; J. P. DEVROEY, op. cit. (n. 52), 1997, p. 954.

${ }_{62}^{62}$ Para el siglo XIII disponemos de una fuente excepcional para conocer la variedad y composición de las dietas monásticas. Se trata del denominado Costumari de Sant Cugat, escrito por el monje Pere Ferrer entre 1221 y 1223, donde se expone con detalle el régimen alimentario de la comunidad. T. DE MONTAGUT (ed.), El costumari del monestir de Sant Cugat del Vallès, Barcelona, 2009.
} 
de acercarnos al régimen alimentario de la comunidad. Sabemos que por aquel entonces el cenobio se encontraba bajo los auspicios de la denominada Regulam sancti Benedicti, aunque ello no nos ofrezca mayor ayuda que la de conocer una norma de comportamiento más o menos cercana (o más o menos alejada) del régimen de vida real de los monjes del monasterio ${ }^{63}$.

Tal y como hemos visto, a lo largo del período estudiado, la comunidad de Sant Cugat del Vallès protagonizó un extraordinario proceso de expansión económica y territorial. De los documentos analizados deducimos que se convirtió en una verdadera señoría jurisdiccional, dotada de numerosos recursos y bien relacionada con los sectores más poderosos de la sociedad. Desconocemos en qué medida tales circunstancias pudieron afectar a las costumbres cotidianas de los monjes. Sabemos que condiciones similares influenciaron, y mucho, a otras grandes abadías europeas, las cuales iniciaron un proceso de relajación de su comportamiento ascético, redundando en una progresiva pérdida de la frugalidad alimentaria exigida por la regla monástica ${ }^{64}$.

Fuera esta desviación de la conducta mayor o menor, debemos suponer que las colaciones monásticas se continuaban realizando en base a los productos obtenidos a través de la explotación de las tierras de su patrimonio. Gracias a los pagos en especie consignados en los documentos podemos saber que al monasterio afluían con asiduidad importantes cantidades de cereal y vino. Más allá de ello, resulta relativamente frecuente la recepción de censos, donaciones piadosas o legados testamentarios en forma de pan (panem o fogacias), sea éste de trigo o de cebada ${ }^{65}$ (fig. 4, mapa 2). Son habituales también las percepciones de otro producto de consumo diario: el vino, "tam de novo quam de vetere"66 (fig. 4, mapa 3). En algunas ocasiones, este tipo de donaciones describen el contenedor utilizado para su entrega al monasterio, como aquellas "II tonas plenas de vino" consignadas en una ejecución testamentaria ${ }^{67}$. Pan y vino constituían la base fundamental de la alimentación monástica medieval.

Por otro lado, el monasterio muestra siempre gran preocupación por el abastecimiento de pescado, alimento imprescindible en la dieta de los monjes (fig. 4, mapa 6).
Ejemplo de tal interés son tanto las piscationes o viveros de peces desarrollados en las presas de los molinos ${ }^{68}$, como la práctica de la pesca en algunos espacios de gran riqueza hidráulica. Así lo vemos en Santa Oliva, cuando se describe un "stagna ad piscandum apta69". De igual manera, la necesidad de abastecerse de pescado se deja entrever también en el conflicto surgido en el año 1013 entre el abad Guitard y Adalaizis por la propiedad de los peces de sus estanques. Dicha contienda acabó dirimiéndose con un reparto salomónico: "expiscari faciant ipsosque pisces communiter iuste dividant per medium"7o.

Todos estos productos (pan, vino, pescado), así como las verduras, hortalizas, legumbres y frutas obtenidas a través de la explotación de su extenso patrimonio debieron ser habituales en las colaciones diarias. Con tal diversidad de productos agrícolas se preparaban ensaladas y menestras apropiadas a su régimen de vida, de las que también disfrutaban quienes se encontraban bajo la hospitalidad del monasterio. Así lo vemos en el año 1107, en la asignación de una renta vitalicia de "libram I panis inter triticum et ordeum et quartanum I de vino et scutulam I pulmento ${ }^{71}$ " al donante de un lote de tierras al monasterio. Es posible que los beneficiarios de este tipo de rentas fueran personas mayores o desvalidas, a las que el monasterio ayudaba con comida (pan, vino y legumbres) y vestido ${ }^{72}$.

Todos los alimentos analizandos hasta el momento constituyen productos permitidos (con restricción de cantidad, en el caso del pan y el vino) tanto por la regla de San Benito de Nursia como por las prescripciones del reformador carolingio Benito de Aniana ${ }^{73}$. Mayor dificultad entraña, sin embargo, la exigencia de numerosos censos en forma de animales vivos o carne (fig. 4, mapa 5). Entre ellos encontramos todo tipo de aves (gallinas, patos, capones) - que las interpretaciones menos rigurosas podían llegar a considerar como un producto apto -, aunque también numerosos animales cuadrúpedos (carneros, corderos) -de consumo radicalmente prohibido entre los monjes sanos. Entre estos últimos destacan, por su abundancia, los que establecen entregas de productos derivados de la carne de cerdo ("obtima perna de porcu"74, "bacconem obtimum ad

${ }_{63}$ Para este período disponemos de muy pocos datos no sólo sobre la alimentación monástica sino también sobre muchos otros aspectos de la vida de la comunidad. Para algunos autores, incluso se pone en duda su condición de monjes, considerándolos clérigos de vida comunitaria bajo jurisdicción de la catedral de Barcelona. A. GARCÍA-SANZ, La noció d'«ordre» en el monaquisme català antic (segles IX-XIII), en II Col-loqui d'historia del monaquisme català, Sant Joan de les Abadesses, 17-20 de noviembre de 1970, vol. II, Abadia de Poblet, 1974, p. 33-37. Para J.M. Salrach, sin embargo, se trataría de una comunidad de posible origen canonical y diocesano a la que durante los siglos IX y X se habría impuesto la regla de San Benito. Se establecería así el principio carolingio de diferenciación entre ordo monasticus y ordo canonicus en un proceso que llevó a muchas comunidades clericales al benedictidismo monástico. J.M. SALRACH, op. cit. (n. 13), p. 129-130. Sobre el programa carolingio de reforma del monacato e imposición de la regla benedictina, véase A. RIERA-MELIS, Alimentació i ascetisme a l'alta edat mitjana. Gènesi de la «dietètica» monàstica benedictina (540-820), en Temps de monestirs. Els monestirs catalans abans de l'any mil, Barcelona, 1999, p. 140-167; G. ARCHETTI, "Mensura victus constituere". Il cibo dei monaci tra Oriente ed Occidente, en L'alimentazione nell'alto medioevo, op. cit. (n. 59), p. 757-797. Entre los documentos más antiguos que mencionan la adscripción de la comunidad santcugatense a la regla de San Benito encontramos CSC, 6, año 913; CSC, 15, año 938; CSC, 53, año 957; CSC, 56, año 958.

${ }^{64}$ A. RIERA-MELIS, op. cit. (n. 39), p. 20.

${ }^{65}$ Así lo vemos en CSC, 792, año 1107. Otras referencias a pan las encontramos en CSC, 50, año 957; CSC, 57, año 959; CSC, 499 , año 1026.

${ }^{66}$ CSC, 499, año 1026. Expresiones similares las encontramos en CSC, 123, año 997.

${ }_{77}^{6}$ CSC, 381, año 1002. Algo similar ocurre en CSC, 526, año 1032 o en CSC, 631, año 1063.

${ }^{68}$ CSC, 130, año 978.

${ }^{69}$ CSC, 449, año 1012.

${ }^{70} \mathrm{CSC}, 452$, año 1013.

${ }^{71}$ CSC, 793, año 1107. Algo similar ocurre en CSC, 791, año 1107.

${ }^{72}$ J.M. SALRACH, op. cit. (n. 13), p. 132.

${ }_{73}$ A. RIERA-MELIS, op. cit. (n. 63), p. 160.

${ }^{74}$ CSC, 461, año 1016 . 
Natale Domini"75). Ello no quiere decir, necesariamente, que los monjes sanos los consumieran. Simplemente significa que afluían al monasterio en forma de censo.

Finalmente, la presencia de carne suína entre las percepciones del monasterio nos lleva a considerar el uso del graso animal en la cocina. Sobretodo si lo relacionamos con la exigüidad de referencias al cultivo de olivos aparecida en el Cartulario, que no llega al o,38\% del total de menciones agrícolas registradas. La documentación estudiada permite documentar únicamente 6 referencias al aceite, de cronología muy tardía ${ }^{76}$, que por su exigüidad constatan el poco aprecio del que disfrutaba este producto por parte del monasterio. Tal vez por ello, tiempo atrás, el monje reformador Benito de Aniana decidiera integrar el lardo en la dieta ordinaria de los monjes benedictinos, obligándoles a renunciar a él los viernes, en Adviento y durante la semana de Quinquagésima77.

\section{CONCLUSIONES}

De acuerdo con el trabajo desarrollado a lo largo de estas páginas creemos poder aportar algunas conclusiones. En primer lugar, y desde un punto de vista metodológico, se demuestra que la perspectiva territorial constituye una magnífica herramienta de aproximación a la complejidad del pasado. El uso de herramientas de georeferenación capaces de representar sobre una misma cartografía digital gran cantidad de datos históricos nos ayuda a interpretarlos de manera conjunta, analizando las relaciones que se establecen entre ellos.

En segundo lugar, y a partir del ejemplo ofrecido por Sant Cugat del Vallès, se pone de manifiesto el interés del análisis territorial en el conocimiento de su trayectoria histórica. A través de estas páginas nos hemos aproximado a la evolución de su patrimonio, a la progresiva creación de espacios de especialización agrícola, a la gestión del patrimonio productivo e incluso a su relación con el mercado. El análisis de todos estos elementos, a su vez, nos ha permitido acercar, no sin dificultades, a un aspecto fundamental de la vida de los monjes: su alimentación, temática sobre la que hasta el momento disponíamos de muy poca información.

Con todo ello se demuestra que la aproximación sistémica que proponíamos el inicio de este trabajo constituye una útil manera de acercarnos al fenómeno monástico medieval. El uso de esta metodología nos ha permitido analizar las relaciones del monasterio con su entorno a partir de distintas aproximaciones: desde el espacio interior (la alimentación de los monjes), el territorio más lejano (la marca extrema del Penedès), pasando por un análisis exhaustivo de su entorno inmediato (el núcleo patrimonial del Vallès). Cada una de estas dimensiones y las relaciones entre ellas constituyen la base de lo que entendemos como paisaje monástico medieval.

\footnotetext{
${ }_{75}$ CSC, 633, año 1063.

${ }^{76}$ CSC, 582, año 1045; CSC, 631, año 1063; CSC, 718, año 1084; CSC, 749, año 1093; CSC, 751, año 1093; CSC, 759, año 1095.

77 A. RIERA-MELIS, op. cit. (n. 63), p. 160.
} 

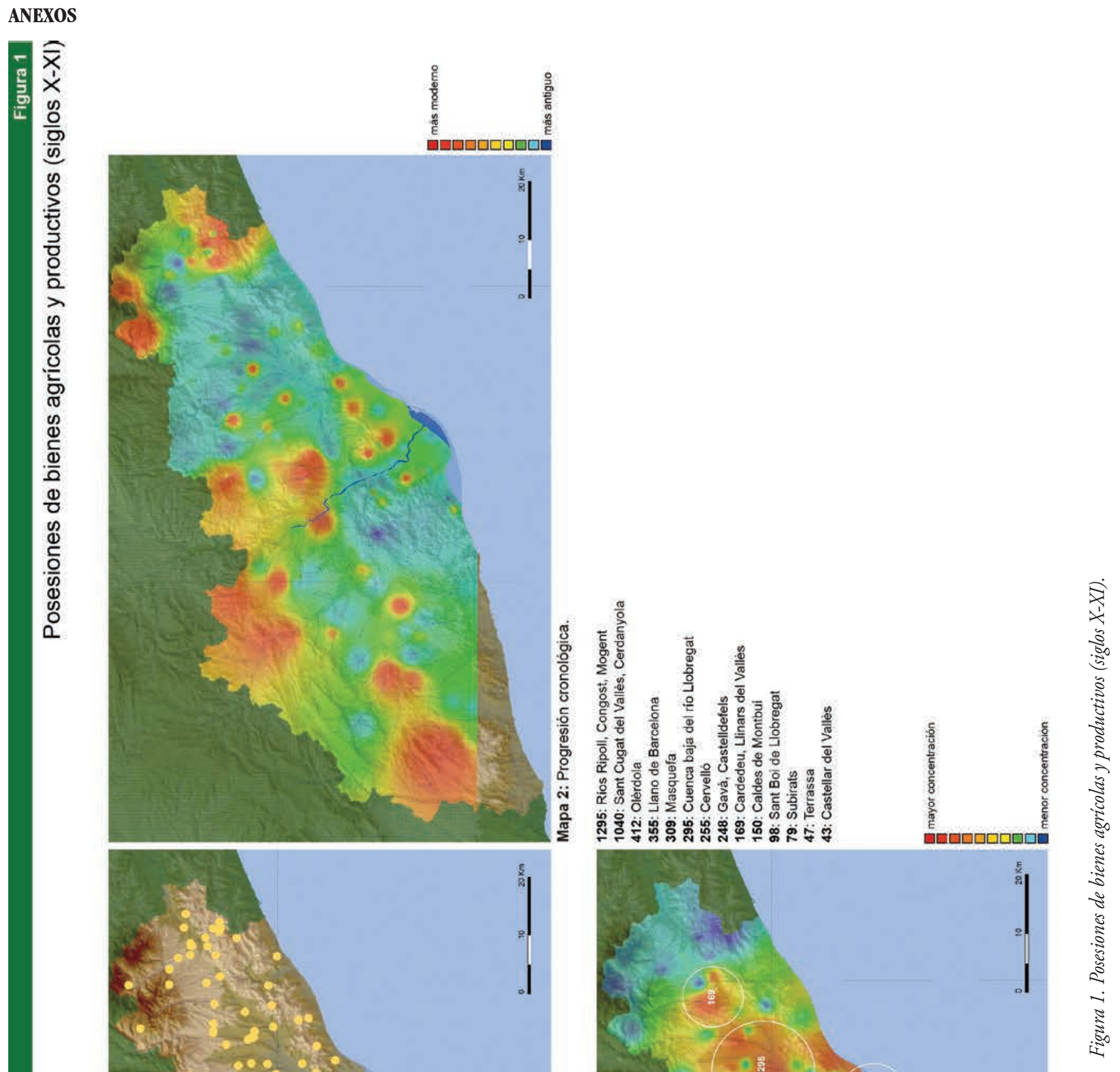

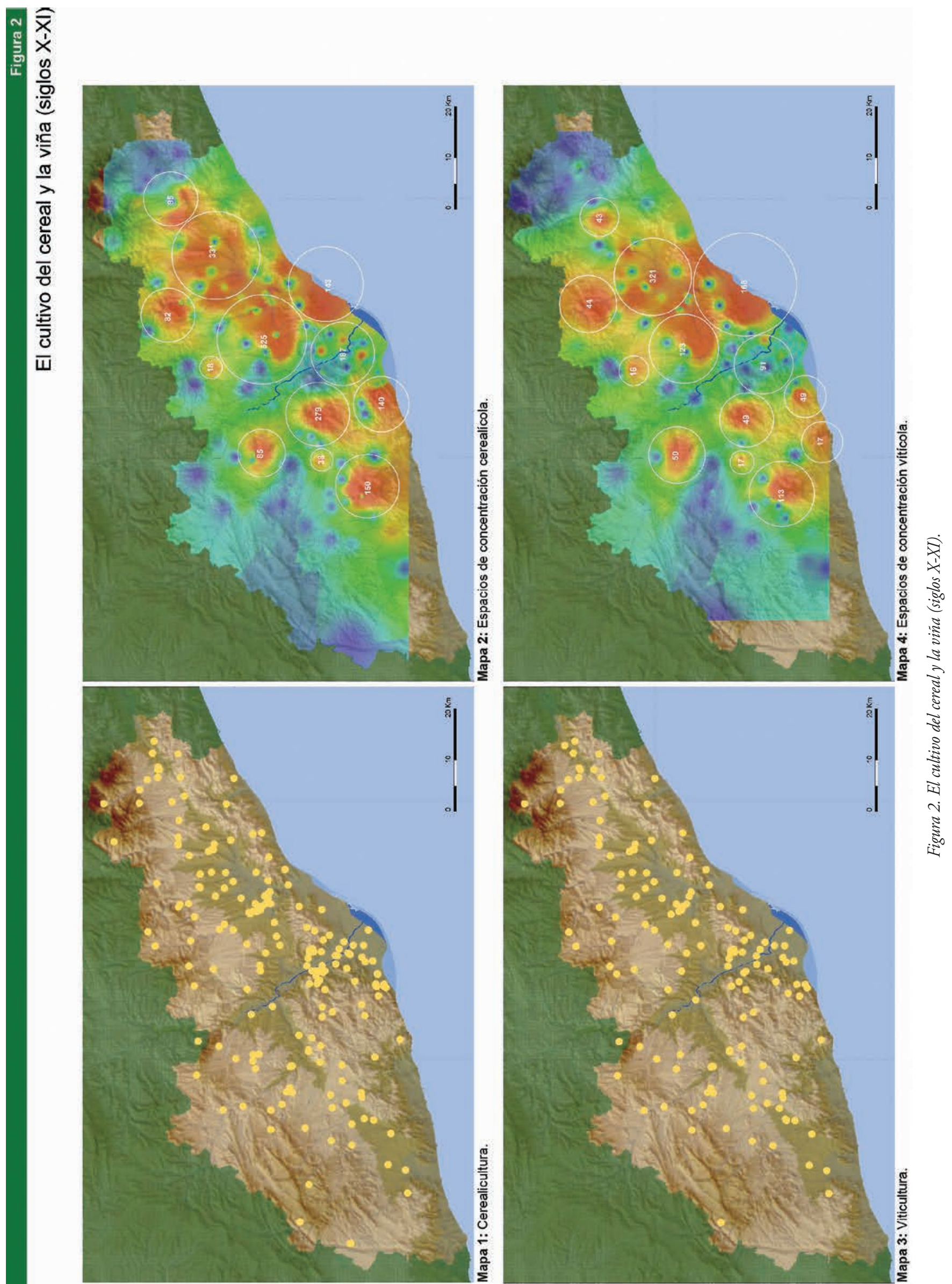

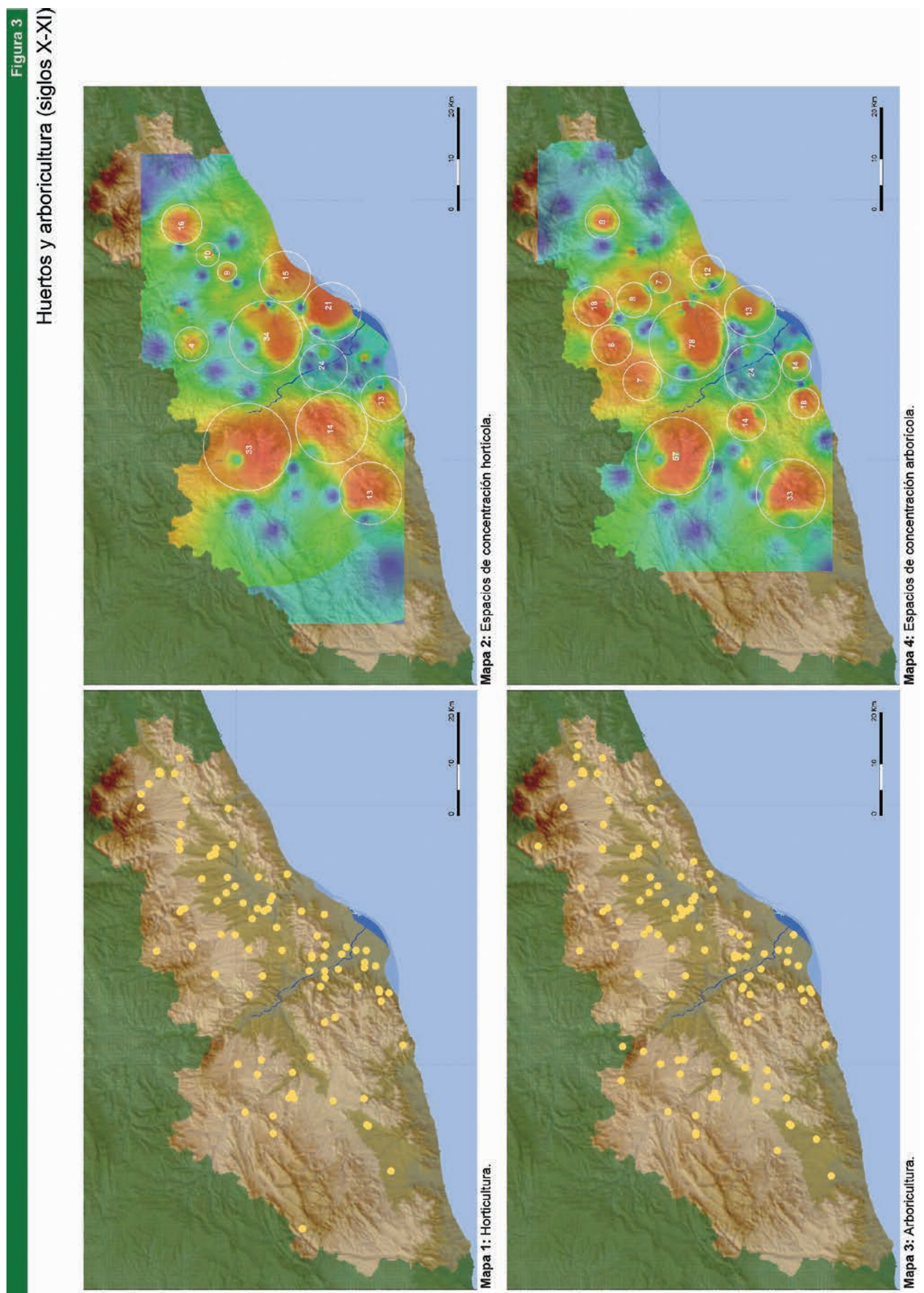

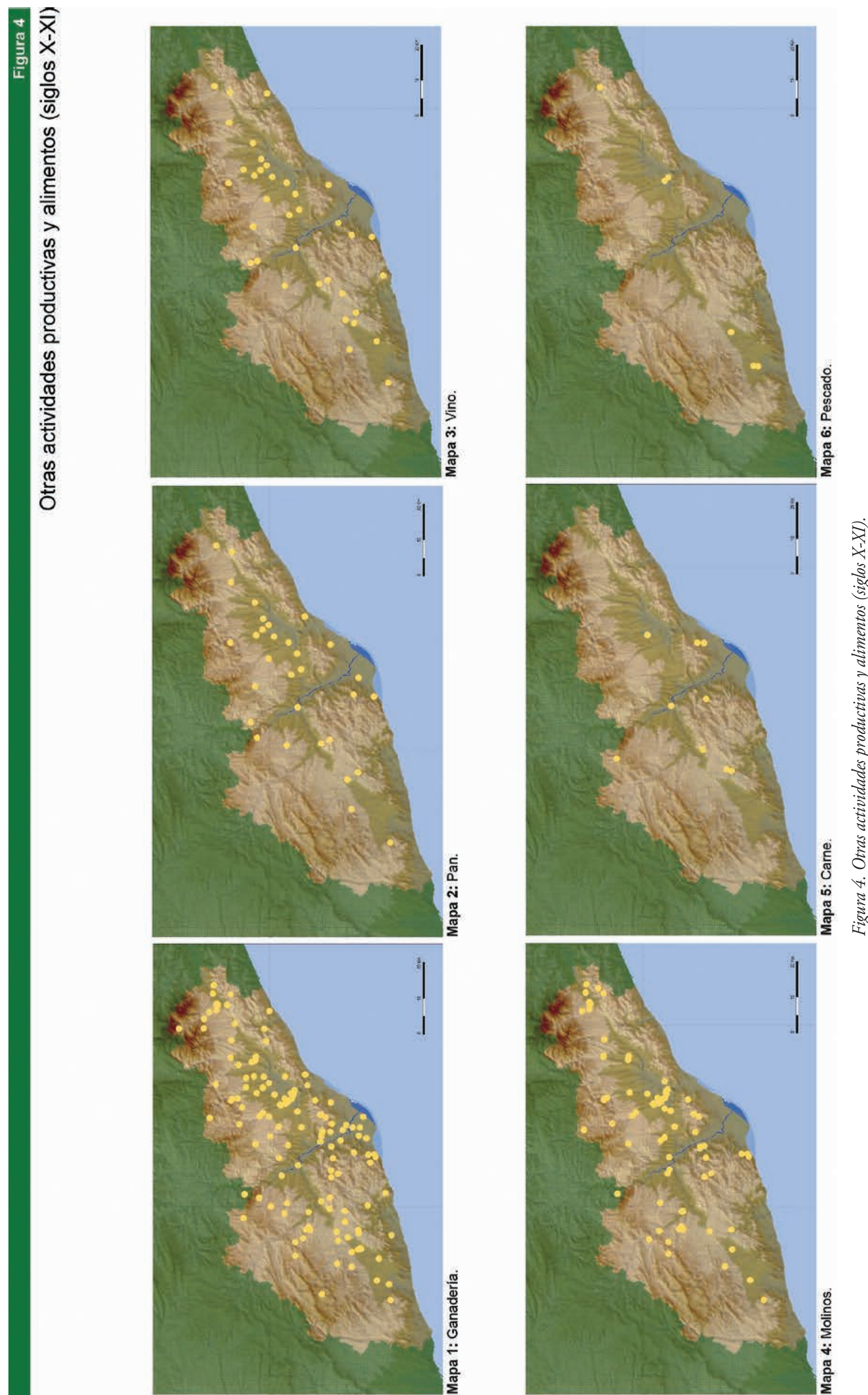